Ann. Zootech., I977, 26 (I), I05-III.

\title{
Note
}

\section{Notes sur l'utilisation des orges françaises dans 1'alimentation des volailles \\ I. - Utilisation de l'orge nue dans l'alimentation de la poule pondeuse}

\author{
J. GUILLAUME \\ avec la collaboration technique de Liliane Derouet, \\ Évelyne Gurlıou et Joëlle GoMez \\ Station de Recherches Avicoles, \\ Centre de Recherches de Tours I.N.R.A. \\ Nouzilly, 37380 Monnaie (France)
}

\section{Résumé}

Nous avons nourri des poules pondeuses commerciales à haut niveau de production à l'aide de régimes contenant 30 ou 60 p. Ioo d'une orge nue d'obtention I.N.R.A. Les performances du lot " $30 \mathrm{p}$. Ioo" sont en tous points comparables à ceux d'un lot de référence à base de maïs. L'utilisation de $60 \mathrm{p}$. Ioo d'orge nue n'abaisse que très faiblement l'intensité de ponte et l'efficacité alimentaire, le poids moyen de l'œuf restant inchangé.

Mesurées sur un échantillon d'orge nue, génétiquement voisine, l'énergie métabolisable de la céréale et la digestibilité de ces protéines s'avèrent nettement supérieures à celles de l'orge vêtue classique.

L'importance des facteurs antinutritionnels (sensu lato) de cette orge est discutée.

\section{Introduction}

L'orge, bien que moins utilisée que le maïs pour l'alimentation de la volaille, présente un grand intérêt économique dans certains pays. Si elle est souvent mal tolérée par le poulet en croissance, elle est beaucoup mieux acceptée par la poule pondeuse (BERG, I959). Cependant, chez l'adulte comme chez le jeune, la valeur énergétique de l'orge classique reste faible (Leong, Jensen et Mc Ginnis, I958, 
Novace k et Petersen, ig67, Sibbald et Slinger, 1963, Göhl et Thom Ke, I976) et on pouvait se demander si les variétés nues (dont les glumelles se séparent de l'amande au cours d'un battage normal) ne présentaient pas, à ce point de vue, un avantage notable.

Si Wilson et Mc NaB, I975, ont obtenu de bons résultats en nourrissant des poulets avec des régimes contenant $75 \mathrm{p}$. Ioo d'orge nue NAKTA, les auteurs qui ont étudié des variétés pourtant similaires dans des conditions voisines ont enregistré de nombreux déboires (ANDERSON, Dobson et WAGSTAFF, I96r, Guillaume et CALET, I973). En fait, la tolérance du poussin vis-à-vis de l'orge semble dépendre essentiellement de la teneur en $\beta$ glucanes et de l'activité $\beta$ glucanasique de la graine ou de la flore intestinale d'une part (RICkEs et al., r962, BuRnETT, r966) et des facteurs antiprotéolytiques d'autre part (LAPORTE et TrÉmolik̀res, I962, Arscott, Hutto et Rachapetayakom, I965. Warchalewski et Skupin, I973), que l'orge soit vêtue ou nue. Comme le poussin paraît plus sensible que l'adulte à ces facteurs antinutritionnels, il nous a paru opportun de rechercher si la poule pondeuse ne pouvait pas constituer un consommateur privilégié de cette nouvelle céréale, les premiers essais en ce sens s'étant avéré encourageants (Guillaume et CALET, r973).

\section{Matériel et méthodes}

Nous avons comparé, chez la poule pondeuse, la valeur alimentaire d'une orge nue expérimentale à celle du maïs en l'incorporant à 2 niveaux : 30 et $60 \mathrm{p}$. Ioo du régime. Cette orge nue provenait de la Station d'Amélioration des Plantes de I'I.N.R.A. à Montpellier; il s'agissait d'une orge d'hiver à deux rangs, elle contenait $88,4 \mathrm{p}$. Ioo de matière sèche et Io,o $\mathrm{p}$. Ioo de protéines $(\mathrm{N} \times 6,25)$. Les poules, d'origine commerciale, étaient de type Rhode Island Red (Warren Studler) et étaient âgées de 30 semaines au début de l'essai. Les 3 régimes, équilibrés, avaient les mêmes teneurs en énergie, protéines et acides aminés limitants. Leur composition figure au tableau $I$.

Les poules ont été réparties selon un dispositif en blocs casualisés de 20 animaux logés 2 par 2 dans Io cages contiguës. On disposait de 40 poules par régime.

Pendant 6 périodes de 4 semaines on a enregistré la ponte, par cage et par jour, la consommation, par bloc et par période, le poids moyen de l'œuf lors de la $4^{e}$ semaine de chaque période ainsi que la mortalité.

A la fin de la $6^{\mathrm{e}}$ période, le stock d'orge nue étant épuisé, nous avons poursuivi l'essai pendant 4 autres semaines en remplaçant l'orge nue par de l'orge vêtue commerciale de type génétique assez voisin : variété RIKA. Pour cela nous avons modifié comme suit la formule de l'aliment "orge 60 ": farine de luzerne 2 p. roo, tourteau de soja " 50 " 3,2 p. roo et huile 3,49 p. Ioo au lieu de 6,o et 4,69 p. Ioo respectivement. Dans le régime "Orge 30 " nous avons conservé, pour chaque constituant, une teneur égale à la moyenne de celle des régimes " soya " et " orge $60 \%$.

Les résultats des 6 premières périodes ont été interprétés après analyse de 
TABLEAU I

Composition des régimes

Composition of diets

\begin{tabular}{|c|c|c|c|}
\hline " & $\begin{array}{c}\text { Soja } \\
(\text { Soyabean })\end{array}$ & $\begin{array}{c}\text { Orge 30 } \\
\text { Barley }\end{array}$ & $\begin{array}{c}\text { Orge } 60 \\
\text { Bayley }\end{array}$ \\
\hline 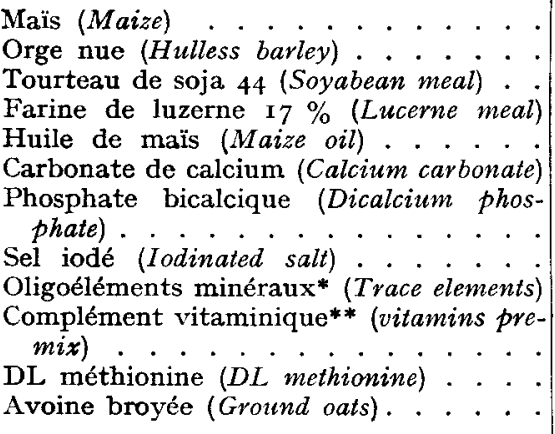 & $\begin{array}{l}59,5 \\
22,0 \\
6,0 \\
2,5 \\
7,45 \\
1,0 \\
0,25 \\
0,10 \\
0,5 \\
0,05 \\
0,65\end{array}$ & $\begin{array}{l}29,75 \\
30,0 \\
21,0 \\
6,0 \\
3,595 \\
7,45 \\
0,975 \\
0,25 \\
0,10 \\
0,5 \\
0,055 \\
0,325\end{array}$ & $\begin{array}{l}60,0 \\
20,0 \\
6,0 \\
4,69 \\
7,45 \\
0,95 \\
0,25 \\
0,10 \\
0,5 \\
0,060 \\
-\end{array}$ \\
\hline
\end{tabular}

\section{Analyse (Analysis) :}

Énergie métabolisable (Kcal/g) (Metabolisable energy) . . . . . . . . . . . . . . 2,79

Protéines $(\mathrm{N} \times 6,25 \%)($ Protein $)$. . . . . . . . . . . . . . . . . . . . . . . $16, \mathbf{1}$

Lysine (Lysine) . . . . . . . . . . . . . . . . . . . . . . . . . . . . . . . 0,83

Méthionine (Methionine) . . . . . . . . . . . . . . . . . . . . . . . . 0,31

Acides aminés soufrés (Sulphur aminoacids) . . . . . . . . . . . . . . . . . . . o,6

Calcium . . . . . . . . . . . . . . . . . . . . . . . . . . . . . . . . . . 3,20

Phosphore disponible (Available phosphorus) . . . . . . . . . . . . . . . . . . 0,30

(*) Apportant en $\mathrm{g}$ pour too $\mathrm{kg}$ d'aliment: cuivre 0 , 14 ; fer $\mathrm{r}, 40$; iode 0,07 ; manganèse 4,33 ; zinc 3,86 ; cobalt o, or 5. (Supplying in g per Ioo kg feed : copper 0.14; iron 1.40 ; iodine 0.07; manganese 4.33; zinc 3.86; cobalt 0.015 .

$\left.{ }^{* *}\right)$ Apportant pour roo $\mathrm{kg}$ d'aliment: vitamine A I 000 ooo UI; vitamine $\mathrm{D}_{3}$ I 00 ooo UI; tocophérol $\alpha_{4} \mathrm{~g}$; thiamine $0,2 \mathrm{~g}$; riboflavine $0,4 \mathrm{~g}$; acide nicotinique $\mathrm{I} \mathrm{g}$; vitamine $\mathrm{K}_{\mathbf{3}} \mathrm{o,2} \mathrm{g}$; pantothénate de calcium $0,4 \mathrm{~g}$; vitamine $\mathrm{B}_{12}$ o,4 mg; avoine q.s.q. $500 \mathrm{~g}$. (Supplying per $100 \mathrm{~kg}$ feed : vitamin $A$ I $000000 \mathrm{IU}$; vitamin $D_{3}$ Ioo ooo $I U$, a tocopherol $4 \mathrm{~g}$; thiamine $0.2 \mathrm{~g}$; riboflavine $0.4 \mathrm{~g}$; niacin $\mathrm{I} \mathrm{g}$; vitamin $\mathrm{K}_{3} 0.2 \mathrm{~g}$; calcium pantothenate $0.4 \mathrm{~g}$; vitamin $B_{12} 0.4 \mathrm{mg}$; oats to $500 \mathrm{~g}$.)

la variance du dispositif en blocs. Pour étudier l'effet du remplacement de l'orge nue par de l'orge vêtue nous avons fait appel à l'analyse de la covariance.

Sur un autre échantillon, nous avons mesuré l'énergie métabolisable chez des coqs adultes par la méthode du ramassage total et la digestibilité des protéines selon TERPSTRA et de HART, I974. Le régime utilisé pour ces mesures contenait 90 p. Ioo d'orge.

\section{Résultats et discussion}

Les principaux résultats figurent au tableau 2. Dans les 3 lots les performances s'avèrent excellentes et la mortalité est négligeable. Le régime contenant $30 \mathrm{p}$. Io , d'orge nue ne diffère en aucun point du régime témoin à base de maīs. A 60 p. Ioo 
TABLEAU 2

Principaux résultats (6 périodes de 4 semaines)

Main results (6 periods of 4 weeks)

\begin{tabular}{|c|c|c|c|c|}
\hline & $\begin{array}{c}\text { Soya } \\
\text { (Soyabean) }\end{array}$ & $\begin{array}{c}\text { O1ge } 30 \\
(\text { Barley } 30)\end{array}$ & $\begin{array}{c}\text { Orge 6o } \\
(\text { Barley } 60)\end{array}$ & $\begin{array}{c}\text { Interpré- } \\
\text { tation } \\
\text { statistique } \\
\text { Statistical } \\
\text { interpre- } \\
\text { tation }\end{array}$ \\
\hline 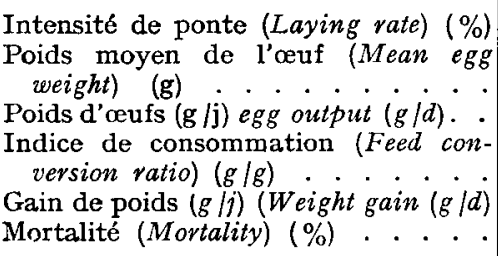 & $\begin{array}{l}84,8 a \\
63,9 \\
55,9 \\
\\
2,31 \\
2,1 \\
o\end{array}$ & $\begin{array}{l}85,7 \quad a \\
64,6 \\
56,9 \\
2,27 \\
2,4 \\
2,5\end{array}$ & $\begin{array}{l}81,3 \quad b \\
63,8 \\
53,9 \\
2,39 \\
3,0 \\
0\end{array}$ & $\begin{array}{c}p<0,05 \\
\text { NS }\end{array}$ \\
\hline
\end{tabular}

d'orge nue le poids de l'œuf demeure inchangé, mais l'intensité de ponte est réduite de façon faible (4 p. 100) mais significative $(p<0,05)$ et l'indice de consommation tend à être un peu plus élevé (3,5 p. roo).

L'évolution en fonction du temps de l'intensité de ponte, tracée sur la figure $\mathrm{I}$, montre que le remplacement de l'orge nue par de l'orge vêtue au cours de la $7^{\mathrm{e}}$ période semble faire disparaître l'infériorité du lot " orge 60 " puisque le changement de ponte de la période 6 à la période 7 est, dans ce lot, significativement distinct de celui que l'on enregistre dans les deux autres $(p<0,05)$.

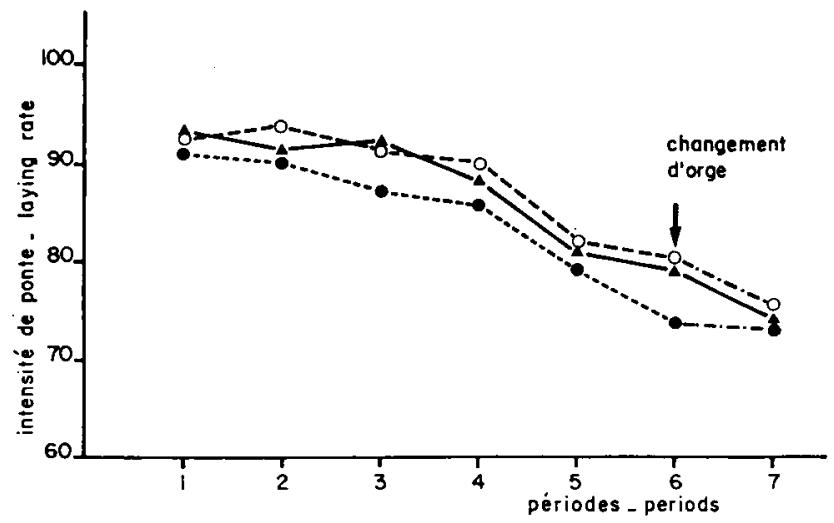

FrG. I. - Intensité de ponte des poules nourries avec les différents régimes.

Laying rate of hens fed experimental diets (at period 6: substitution of normal barley for nacked barley.

- - $\perp$ Témoin maïs (Maize Control)

- ……...... - Orge 60\% (Barley 60\%)

- …......... O Orge $30 \%$ (Barley $30 \%$ ) 
Il semble donc que, à 60 p. Ioo du régime, l'orge nue éprouvée est un peu moins bien tolérée par la Poule que l'orge vêtue utilisée dans cet essai; aucune conclusion définitive ne peut toutefois en être tirée, la durée de l'expérience sur orge vêtue étant très brève et les échantillons n'ayant pas été cultivés dans les mêmes conditions. Il n'est pas non plus possible d'expliquer les causes de la légère diminution des performances des poules nourries avec $60 \mathrm{p}$. Ioo d'orge nue : il peut s'agir d'une conséquence des glucides indésirables ou des activités $\beta$ glucanasiques ou antiprotéolytiques; la présence éventuelle de tannins ${ }^{(}{ }^{\mathbf{1}}$ ) pourrait également, à une certaine dose, réduire l'utilisation digestive de la ration (GHöL et THомке, I976). Il serait donc opportun d'effectuer des analyses plus poussées et des mesures d'activités enzymatiques avant de reprendre l'étude de la valeur alimentaire de ce type d'orge chez la volaille.

Le seul pas fait dans cette direction : la mesure de l'énergie métabolisable et de la digestibilité des protéines - effectuée sur un échantillon génétiquement voisin mais non identique - a donné les valeurs suivantes :

énergie métabolisable : $3,62 \mathrm{kcal} / \mathrm{g}$ de matière sèche,

$8 \mathrm{r}, 6$ p. roo de l'énergie brute,

coefficient de digestibilité apparent des protéines : 78,2 p. Ioo.

La valeur énergétique de l'échantillon utilisé est donc supérieure de $20 \mathrm{p}$. Ioo environ à la valeur moyenne des orges vêtues telle que l'ont trouvée les auteurs cités dans l'introduction. Rappelons que sur un autre échantillon d'orge nue, nous avions trouvé (GUillaume et CALET, I973) une différence de valeur énergétique de ro p. roo par rapport à un échantillon d'orge vêtue cultivée dans les mêmes conditions, mais d'excellente qualité.

La digestibilité des protéines est également supérieure à celle de l’orge vêtue mesurée par Potter, Stutz et Matterson, I965, Vogt et Stute, i971, et nousmême (Guillatme, i976).

\section{Conclusion}

L'orge nue possède donc chez la volaille adulte une valeur nutritive comparable à celle du blé. Elle est très bien utilisée par la poule pondeuse de haute productivité, bien qu'une très légère baisse de la production apparaisse quand on l'emploie comme seule source de céréale. En revanche, même à ce niveau, elle ne s'avère pas inférieure au maïs en ce qui concerne le poids moyen de l'œuf comme l'ont trouvé de nombreux auteurs qui attribuent au maïs un facteur inconnu nécessaire à l'obtention du poids d'œuf maximal (JENSEN et al., I958, BERG, I959). On peut donc recommander l'utilisation de cette orge dans les aliments pour poule pondeuse jusqu'à une dose représentant 30 p. Ioo des céréales.

Reçu pour publication en octobre 1976.

(1) Une partie des grains de l'échantillon d'orge utilisée contenait des tannins mis en évidence par la méthode décrite par Martin-Tanguy, Guillaume et Kossa, i977, J. of Sci. Food Agric. (sous. presse). 


\section{Remerciements}

Nous remercions M. J. Cherry de la Station d'Amélioration des Plantes de l'I.N.R.A., de Montpellier pour la fourniture gratuite de l'orge utilisée.

\section{Summary}

\section{Utilisation of French barley in poultry feeding. I. - Hulless barley in laying hen rations}

Laying hens of commercial origin with a high production level were fed diets containing $3^{\circ}$ or $60 \%$ hulless barley (I.N.R.A. variety). Overall performances of the " $30 \%$ " group were similar to those of the control group receiving maize. Use of $60 \%$ hulless barley only slightly reduced the laying rate and feed efficiency, while the mean egg weight remained unchanged.

According to measurements made on a genetically related sample of hulless barley, metabolisable energy and protein digestibility proved to be definitely higher in hulless than in regular barley.

The importance of the antinutritional (sensu lato) factors of this barley is discussed.

\section{Références bibliographiques}

Amderson J. J., Dobson D. C., Wagstaff R. K., 196r. Studies of hulless barley in chick diets and means of increasing this value. Poult. Sci., 40, I57I-I584.

Arscott G. H., Hutto D. C., Rachapetayakom P., 1965. Use of barley in high efficiency broiler rations -7 - Pancreatic enlargment in chicks fed barley containing diets. Poult. Sci., 44. $432-434$.

Berg L. R., 1959. Enzyme supplementation of barley diets for laying hens. Poult. Sci., 38, II $32-1139$.

BURNETt G. S., I966. Studies of viscosity as the probable factor involved in the improvement of certain barleys for chickens by enzyme supplementation. Brit. Poult. Sci., 7, 55-75.

GöнL B., Тном ке S., 1976. Digestibility coefficient and metabolizable energy of barley diets for layers as influenced by geographical area of production. Article non publié. (Université d'Uppsala, Suède.)

Guinlaume J., Calet C., r973. Orge et avoine nues dans l'alimentation du poulet et de la poule pondeuse. J. Rech. Avic. cunic., Paris déc. 73, I73-I 75 (publ. I.T.A.V.I., 28, rue du Rocher, 75009 Paris).

GUILlAUME J., I976. Notes sur l'utilisation des orges françaises dans l'alimentation des volailles. II. Valeurs énergétiques et digestibilité des protéines selon la variété et le lieu de culture (A paraittre).

Jensen L. S., Allred J. B., Fry R. E., McGinnis J. M., I958. Evidence for an unidentified factor necessary for maximum egg weight in chickens. J. Nutr., 65, $219-233$.

Laporte J., Tremolik̀res J., 1962. Action inhibitrice des farines de tiz, d'avoine, de mais, d'orge, de blé, de seigle, de sarrasin sur certains enzymes protéolytiques du pancréas. C.R. Soc. Biol., 156, I 26r-1263.

Leong K. C., Jensen L. S., McGrnnis J., i958. Effect of water treatment and fungal enzyme addition on metabolizable energy of pearley barley. Poult. Sci., 37, r220-1221 (Abstr.).

Novacek E. J., Petersen C. F., 1967. Metabolizable energy of the anatomical parts and other fractions of western barley and the effect of enzymes and water treatment. Poult. Sci., 46, I $008-1015$.

Potter L. M., Stutz M. W., Matterson L. D., 1965. Metabolisable energy and digestibility coefficients of barley for chicks as influenced by water treatment or by the presence of fungal enzymes. Poult Sci., 44, 565-573. 
Rickes E. L., Ham E. A., Mascatelli E. A., Ott W. H., i962. The isolation and biological properties of a $\beta$ glucanase from B. subtilis. Arch. Biochem. Biophys., 96, 371-375.

Sibbald I. R., Slinger S. T., I963. Nutritive value of ten samples of western canadian grains. Poult. Sci., 42, $276-277$.

Terpstra K., DE HART M., I974. The estimation of urinary nitrogen and fecal nitrogen in poultry excreta. Tierphysiol. Tievernahrung Futtermitelk., 32, 306-320.

Vogt H., Stute K., I97I. Uber der Verdaulichkeit einiger Kohlenhydratfraktionen (Zucker, Stärke, Pentosane, Rohcellulose, Lignin) in Hühnerfutter. Arch Geflkde., 35, $29-35$.

WARChalewski J. R., SKUPIN J., 1973. Isolation and properties of trypsin and chymotrypsin inhibitors from barley grits after storage. J. Sci. food agric., 24, 995-Ioro.

Wilson B. J., McNab J. M., I975. Diets containing conventional, naked and high amylose barleys for broilers. Brit. Poult. Sci, 16, 497-504. 